Journal of Engineering and Applied Sciences 14 (Supplimentary 01): 8519-8522, 2019

ISSN: 1816-949X

(C) Medwell Journals, 2019

\title{
Spectral Matching Algorithm for Horticulture Application
}

\author{
R. Sowmya and Shivani Deota \\ Manipal Academy of Higher Education, Deptartment of Electrical and Electronics, \\ Manipal Institute of Technology, Manipal, India, sowmya.r@manipal.edu, 08970507444
}

\begin{abstract}
Horticulture lighting industry is one of the fastest growing industries which is capable of overcoming the limitations of changing light conditions around the year. Plants need a band of wavelengths which drives photosynthesis and aids growth. The research aims to find an optimal solution for the spectrum matching function. Two algorithms have been discussed for optimal spectral matching. The first optimization algorithm for n-channel LED was simulated, however; validation for the same is needed before going to real time design. In the second method, a database of LED along with curve-fit tool and Gaussian model were used in the simulation. Results indicated a larger database of LEDs will lead to better spectrum match.
\end{abstract}

Key words: LED, spectral matching algorithm, Gaussian model, horticulture, optimization, simulation

\section{INTRODUCTION}

Horticulture lighting is one of the fastest growing application, since, it can overcome the limitations of changing weather and provide the required vegetation around the year. HPS was most commonly used as artificial light source with other conventional light sources in horticulture industry.

But with time the growers and farmers were expecting for a single light source which could be used for different applications, increase in efficiency and reduce the power consumption and expect longer lifetime of LEDs (Anonymous, 2018a, b). On the other hand, Light Emitting Diodes (LEDs) are the latest light source used due to its advantages over traditional light source as they are available in all the wavelengths in visible range can be controlled and tuned, power consumption is less and many more (Radetsk, 2018). The light perceived by humans is different than plants and are sensitive to different wavelengths compared to humans. Photo synthetically Active Region (PAR) is a range from $400-700 \mathrm{~nm}$ where the process of photosynthesis is carried out. Red and blue light plays important role in achieving proper photosynthesis and help plant growth and its development. They can replace the fluorescent spectrum of continuous spectrum. Mainly, approximately $600-700 \mathrm{~nm}$ of red-orange light and $400-500 \mathrm{~nm}$ of blue-violet light are peak absorption wavelength (Wenger et al., 2003; Massa et al., 2008).

Spectral characteristics are the important characteristics while designing a tunable light source. This research aims to obtain a spectral match according to the desired spectrum given by the user for particular application (Burgos-Fernandez et al., 2016; Atia et al., 2018). Different methods and algorithms for obtaining the spectral match are mentioned in the below sections and each with its advantages and disadvantages (Hu et al., 2014; Gan et al., 2015; Fryc et al., 2005).

Tunable light sources for better plant growth which could not be achieved with conventional light sources. Controlling of light according to the user requirement with required wavelength for the growing process of the plants can be more beneficial and effective way of using a light source (Fryc et al., 2004; Yao, 2016).

\section{MATERIALS AND METHODS}

Two different methods were used to find the best spectral match with the desired spectrum. First method is to find the optimal algorithm for n-channel LEDs and the second method is based on multi-term Gaussian Model (Tian, 2016; Lukovic et al., 2016).

Optimization algorithm: Develop a database of $n$ types of color LEDs with given parameters. The aim is to find optimal solution for spectral match with minimum error. For ith color LED, let $\Lambda$ denote the wavelength $(\Lambda)$ denote the SPD, denote the weight, $\mathrm{I}(\Lambda)$ denotes the SPD for the target spectrum. Then the minimization function can be written as our objective function:

$$
\min [\mathrm{f}(\mathrm{w})]=\left(\sum_{400}^{700}\left(\sum_{\mathrm{i}=0}^{\mathrm{n}} \mathrm{w}_{\mathrm{i}} \mathrm{P}_{\mathrm{i}}(\kappa)-1(\kappa)\right)^{2}\right)
$$

Corresponding Author: R. Sowmya, Manipal Academy of Higher Education, Deptartment of Electrical and Electronics, Manipal Institute of Technology, Manipal, India, sowmya.r@manipal.edu, 08970507444 
With constraints as $w_{i} \geq 0$. This algorithm is based on SIM (Spectral Illuminant Matching) (LeGendre et al., 2017) which allows us to obtain a spectrum which tries to the match the target spectrum. The interior point method searches for $n$ weighting values $\left(\mathrm{w}_{\mathrm{i}}\right)$ to be applied to each n spectral channels $P_{i}(\Lambda)$ which composes the proposed light source to resemble the standard illuminant. This algorithm stops when it finds a minimum value. Namely $\mathrm{I}(\Lambda)$ is a $\mathrm{m} \times 1$ vector representing the $\mathrm{m}$ sampling points over $400-700 \mathrm{~nm}$ range in steps of $5 \mathrm{~nm}$ of the real illuminant. $P_{i}(K)$ is a $m \times n$ matrix that indicates $n$ spectra of the LEDs sampled at $m$ points, $w_{i}$ is a $n \times 1$ vector containing $n$ weighting values. This algorithm was implemented using excel solver.

And this can be solved using MATLAB function fmincon which attempts to find a constrained minimum of a scalar function of one or several variables.

Simulation with multi-term Gaussian model: In this part, to simulate the required optimized spectra for general application of plants a multi-term Gaussian model with MATLAB optimization toolbox is used. The function is shown in Eq. 2. Simulations with two, four, six, eight term Gaussian model are possible. Initially approximate each LED's SPD using a Gaussian function:

$$
\mathrm{SPD}=\mathrm{a} * \mathrm{e}^{-\mid\left(\frac{\left(1-\kappa_{\mathrm{P}}\right)}{\mathrm{w}}\right)^{2}}
$$

The LED spectrum depends on the peak amplitude a, peak wavelength $\hat{R}_{\mathrm{p}}(\mathrm{nm})$ and full width at half maximum $\mathrm{w}(\mathrm{nm})$ which is $\widehat{K}_{\text {FWFM }}(\mathrm{nm})$. Equation 2 can be written as Eq. 3:

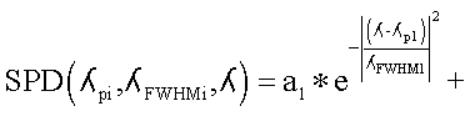

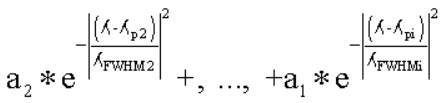

For i number of terms where $\mathrm{i}=1,2,3, \ldots$,

\section{RESULTS AND DISCUSSION}

Figure 1 shows the target spectrum considered as our sample SPD here has been measured using the integrating sphere. For plants the important phenomenon is photosynthesis and they have different range of wavelengths from $400-700 \mathrm{~nm}$ called PAR-photosynthetically active region for that to occur.

Using optimization algorithm: According to Eq. 1, the collected data for spectral match from (420-480 nm)-red and (620-680 nm)-blue, since, they have greater impact on the plants was chosen and SPDs were simulated (Fig. 2)

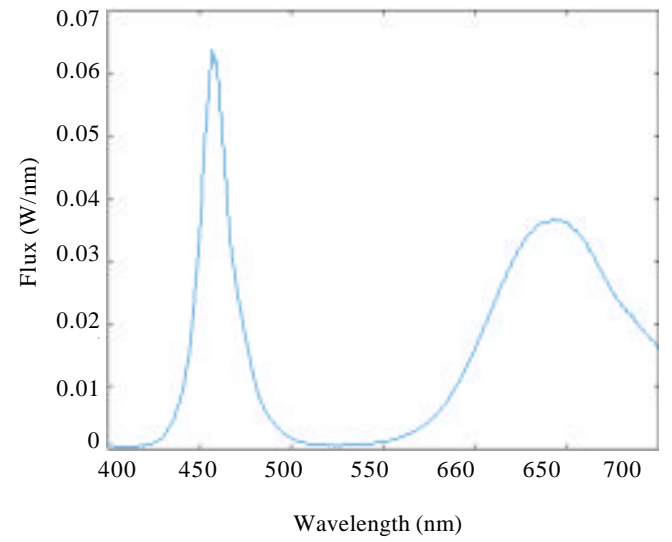

Fig. 1: SPD of the target light source

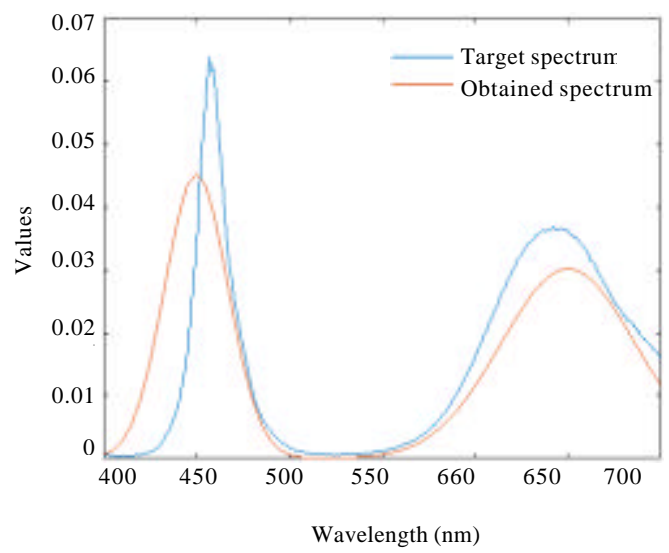

Fig. 2: Using optimization algorithm

Assuming $\mathrm{n}=2$ (taking average for red and blue SPD) and ratio of B:R LEDs to be 1.5 using the optimal algorithm mentioned in section 7.3, Eq. 2, we use the excel solver to solve the problem which is described in Section 8. The weights found were, $\mathrm{w}_{1}=0.05$ and $\mathrm{w}_{2}=0.03$ and the minimum error RMSE was 0.0046 .

Therefore, number of LEDs needed would be aproximately 5 blue and 3 red LEDs. From Fig. 3, we can see that the spectrum of obtained light is not exactly matching the spectrum of the target light source, this means, we need to add more led channels to this spectrum to perfectly match the target.

Using simulation with multi-term Gaussian model: The $x$ data and $y$ data is loaded in the curvefit toolbox of the matlab and changing the model type to Gaussian and entering the number of terms as desired, we can obtain the coefficient values. First 2 term model was introduced but with that the FWHM value was too large which is practically difficult to get from the manufactures. 


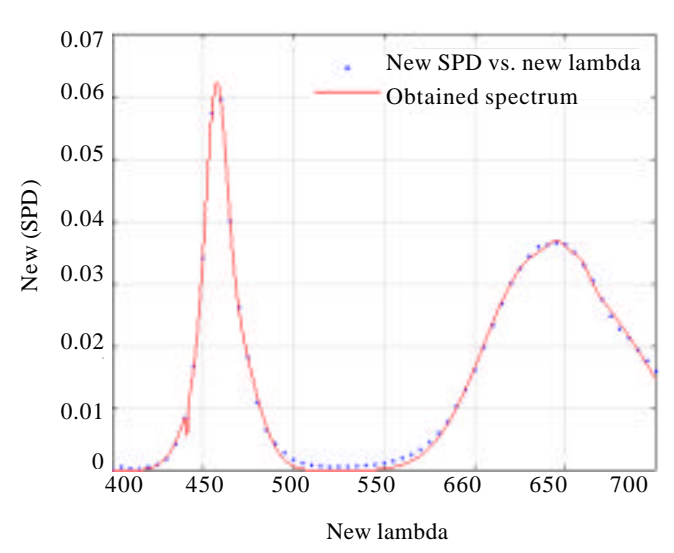

Fig. 3: Spectral matching using curve-fit tool (8-term)

Table 1: Values of FWHM, peak wavelength and coefficients for 8-term Gaussian model

\begin{tabular}{lcll}
\hline No. of terms. & Peak wavelength & FWHM & Co-efficients \\
\hline 1 & 457 & 7.817 & 0.03618 \\
2 & 646 & 8.0874 & 0.002518 \\
3 & 624 & $4.189 \mathrm{e}^{-05}$ & 0 \\
4 & 658 & 6.135 & 0.002355 \\
5 & 441 & 0.753 & -0.004603 \\
6 & 626 & 36.52 & 0.02586 \\
7 & 672 & 43.6 & 0.02124 \\
8 & 461 & 20.1 & 0.02717 \\
\hline Goodness of fit: SSE: $2.256 \mathrm{e}^{-05 ;} \mathrm{R}^{2}:$ & $0.9984 ;$ Adjusted $\mathrm{R}^{2}:$ & $0.9974 ;$ RMSE: \\
0.0007809 &
\end{tabular}

So with increasing number of terms, we can reduce the FWHM value and also match the spectrum. So, for 8-term Gaussian model the following data is obtained. So, from Table 1, we can conclude that LEDs with approximately available peak wavelength from 457,461 , 626,646 and $672 \mathrm{~nm}$ can be used to obtain our target spectrum. Still, we can see FWHM value to be more, so, further 12-term Gaussian model can be introduced to reduce the values. We can further validate this results with the market available LEDs theoretically and practically.

\section{CONCLUSION}

We have shown two methods here for finding the optimal spectral match. The first one is using optimization algorithm for 2-channel LED but we can see that the spectral match obtained is not proper and we need to add more channel of LEDs. In the second method, we have used curve-fit tool and used Gaussian model to find the solution. Using this, we obtained the results as database for FWHM, Peak wavelength and weights to spectrally match the target source. FWHM is expected to be $<20 \mathrm{~nm}$ for practical availability of the LEDs. A theoretical study is presented for the same. The ratio of red to blue LEDs is also defined to attain the spectral match. Using this, we can find other quantities such as PPF, CCT, YPF, efficiency. So, from this, we can say that if we increase the number of LED channels and keep the FWHM narrow that is $\leq 20 \mathrm{~nm}$, we can achieve the spectral match for the target source.

\section{SIGNIFICANCE STATEMENT}

Horticulture is a developing area which has potential to meet the demand for food scarcity. Supplementary lighting plays an important role in the growth and development of plants. The optimization of LEDs is a prime factor that contributes to energy savings.

\section{ACKNOWLEDGEMENT}

I am grateful to Department of Electrical and Electronics, Manipal Institute of Technology for their support.

\section{REFERENCES}

Anonymous, 2018a. Designing for LED horticulture applications with proper circuit protection. Littelfuse, Inc., Chicago, Illinois, USA. https://www. littelfuse.com/ /media/electronics/application_notes/ littelfuse_horticulture_application_note.pdf.pdf

Anonymous, 2018b. LED lighting systems for horticulture applications. Illumitex, Austin, Texas, USA.

Atia, D.M., H.T. El-Madany, A. Eliwa, A. Samir and M. Zahran et al., 2018. Spectral irradiance estimation of light emitting diode solar simulator based on genetic algorithm. Res. J. Appl. Sci. Eng. Technol., 15: 227-235.

Burgos-Fernandez, F.J., M. Vilaseca, E. Perales, J.A. Herrera-Ramirez and F.M. Martinez-Verdu et al., 2016. Spectrally tunable light source based on light-emitting diodes for custom lighting solutions. Opt. Appl., 46: 118-129.

Fryc, I., S.W. Brown and Y. Ohno, 2005. Spectral matching with an LED-based spectrally tunable light source. Proceedings of the 5th International Conference on Solid State Lighting Vol. 5941, September 14, 2005, San Diego, California, USA., pp: 300-308.

Fryc, I., S.W. Brown, G.P. Eppeldauer and Y. Ohno, 2004. A spectrally tunable solid-state source for radiometric, photometric and colorimetric applications. Proceedings of the 4th International Conference on Solid State Lighting Vol. 5530, October 20, 2004, Denver, Colorado, USA., pp: 150-159. 
Gan, R.T., Z.N. Guo and J.B. Lin, 2015. Spectral matching technology for light-emitting diode-based jaundice photodynamic therapy device. J. Mod. Opt., 62: 212-217.

Hu, N.C., Y.C. Feng, C.C. Wu and S.L. Hsiao, 2014. Optimal radiant flux selection for multi-channel light-emitting diodes for spectrum-tunable lighting. Light. Res. Technol., 46: 434-452.

LeGendre, C., X. Yu and P. Debevec, 2017. Optimal LED selection for multispectral lighting reproduction. Electron. Imaging, 2017: 25-32.

Lukovic, M., V. Lukovic, I. Belca, B. Kasalica and I. Stanimirovic et al., 2016. LED-based Vis-NIR spectrally tunable light source-the optimization algorithm. J. Eur. Opt. Soc. Rapid Publ., 12: 1-12.

Massa, G.D., H.H. Kim, R.M. Wheeler and C.A. Mitchell, 2008. Plant productivity in response to LED lighting. Hort Sci., 43: 1951-1956.
Radetsk, L.C., 2018. LED and HID horticultural luminaire testing report. Learning Resource Centre, London, UK. https://www.lrc.rpi.edu/programs/energy/ pdf/HorticulturalLightingReport-Final.pdf

Tian, F., 2016. Study and optimization of lighting systems for plant growth in a controlled environment. Ph.D Thesis, Paul Sabatier University, Toulouse, France.

Wenger, A., T. Hawkins and P. Debevec, 2003. Optimizing color matching in a lighting reproduction system for complex subject and illuminant spectra. Proceedings of the 14th Eurographics Workshop on Rendering, June 25-27, 2003, Leuven, Belgium, pp: 249-259.

Yao, Q., 2016. Application-dependent spectrum optimization of four-package LEDs. Light. Res. Technol., 48: 844-856. 